\title{
Checklist para indicação de ventilação não invasiva em idosos pós-acidente vascular cerebral
}

\author{
Checklist for indication of non-invasive ventilation in elderly post-cerebral vascular accident \\ Checklist para la indicación de ventilación no invasiva en posibles accidentes vasculares cerebrales
} ancianos

\author{
Maria Lucrécia de Aquino Gouveia \\ ORCID: https://orcid.org/0000-0002-2542-258X \\ Universidade Federal da Paraíba, Brasil \\ E-mail: lucreciagouveia@yahoo.com.br \\ Ana Karênina de Freitas Jordão do Amaral \\ ORCID: https://orcid.org/0000-0002-7470-7717 \\ Universidade Federal da Paraíba, Brasil \\ E-mail: akfjafono@hotmail.com \\ Rita de Cassia Marchi Barcello Dalri \\ ORCID: https://orcid.org/0000-0002-6575-5426 \\ Universidade de São Paulo, Brasil \\ E-mail: ritacmbdalri@bol.com.br \\ Antônia Lêda Oliveira Silva \\ ORCID: https://orcid.org/0000-0001-7758-2035 \\ Universidade Federal da Paraíba, Brasil \\ E-mail: alfaleda2@gmail.com
}

\begin{abstract}
Resumo
Objetivo: Construir e validar um checklist para indicação de ventilação não invasiva em idosos pós-acidente vascular cerebral. Método: Estudo metodológico realizado de outubro a novembro de 2017 em um hospital geral do Estado da Paraíba, Brasil. Foram instituídos dois grupos de participantes, sendo o primeiro denominado GD (grupo de doutores e experts em fisioterapia respiratória) e o segundo GF (grupo de fisioterapeutas atuantes em assistência cardiorrespiratória com pessoas idosas), sendo tais grupos compostos por 10 participantes cada. Após a emissão dos pareceres dos participantes do GD e do GF, foi elaborado o checklist. Resultados: Existe viabilidade de aplicação clínica do checklist e este pode auxiliar nas tomadas de decisões. Sua relevância foi demonstrada por meio de significância estatística ( $\mathrm{p}=0,007)$, com possibilidade de aplicação em curto tempo ( $\mathrm{p}=0,0001)$; quanto a abrangência de conteúdo, 50\% dos participantes $(\mathrm{n}=10)$ relataram que o checklist é abrangente e $50 \%(\mathrm{n}=10)$ afirmaram que é necessário acréscimo de conteúdo; todos os participantes foram unânimes que o instrumento tem boa apresentação, é objetivo, coerente e claro em seu conteúdo $(n=20 ; 100 \%)$. Conclusão: Observou-se a importância do instrumento na tomada de decisão na indicação de ventilação não invasiva em idosos pós-acidente vascular cerebral. O checklist é um recurso tecnológico que contribui para esta tomada de decisão, facilita a prática profissional e serve como base para novas políticas públicas, pois proporciona benefícios à saúde da pessoa idosa, além de gerar menor tempo de internação e evitar a piora no quadro do idoso pós-acidente vascular cerebral.
\end{abstract}

Palavras-chave: Checklist; Idosos; Acidente vascular cerebral; Respiração com pressão positiva; Ventilação não invasiva.

\begin{abstract}
Objective: Constructing and validating a checklist for indication of noninvasive ventilation in elderly after stroke. Method: A methodological study conducted from October to November 2017 in a general hospital in the State of Paraiba, Brazil. Two groups of participants were established, the first called GD (group of doctors and experts in respiratory physiotherapy) and the second GP (group of physiotherapists working in cardiorespiratory care with the old), and these groups were composed of 10 participants each. After issuing the opinions of the GD and GP participants, the checklist was prepared. Results: There is feasibility of clinical application of the checklist, and this can assist in decision making. Its relevance was demonstrated by statistical significance $(\mathrm{p}=0.007)$, with the possibility of application in a short time $(\mathrm{p}=0.0001)$; regarding the content coverage, $50 \%$ of the participants $(\mathrm{n}=10)$ reported that the checklist is comprehensive and $50 \%(\mathrm{n}=10)$ stated that it is necessary to increase content; all participants were unanimous that the instrument has good presentation, is objective, coherent and clear in its content $(n=20 ; 100 \%)$. Conclusion: The importance of the instrument in decision-making in the indication of noninvasive ventilation in elderly after stroke was observed. The checklist is a technological resource that contributes to this decision-making, makes professional
\end{abstract}


practice easier and serves as a basis for new public policies, as it provides benefits to the health of the elderly, besides generating shorter hospitalization time and avoiding worsening in the situation of the elderly after stroke.

Keywords: Checklist; Stroke; Breathing with positive pressure; Noninvasive ventilation.

\section{Resumen}

Objetivo: Construir y validar una lista de verificación para la indicación de ventilación no invasiva en ancianos después del accidente cerebrovascular. Método: Estudio metodológico realizado de octubre a noviembre de 2017 en un hospital general del Estado de Paraíba, Brasil. Se establecieron dos grupos de participantes, el primero denominado GM (grupo de médicos y expertos en fisioterapia respiratoria) y el segundo GF (grupo de fisioterapeutas que trabajan en cuidados cardiorrespiratorios con los ancianos), y estos grupos estuvieron compuestos por 10 participantes cada uno. Después de emitir las opiniones de los participantes de GM y GF, se preparó la lista de verificación. Resultados: Existe la viabilidad de la aplicación clínica de la lista de verificación y esto puede ayudar en la toma de decisiones. Su relevancia fue demostrada por significación estadística ( $\mathrm{p}=0,007)$, con posibilidad de aplicación en poco tiempo ( $\mathrm{p}=0,0001)$; en cuanto a la cobertura de contenido, $50 \%$ de los participantes $(\mathrm{n}=10)$ relataron que la lista de verificación es comprensiva y $50 \%$ $(n=10)$ afirmaron que es necesario aumentar el contenido; todos los participantes fueron unánimes en que el instrumento tiene buena presentación, es objetivo, coherente y claro en su contenido $(n=20 ; 100 \%)$. Conclusión: Se observó la importancia del instrumento en la toma de decisiones en la indicación de ventilación no invasiva en ancianos después del accidente cerebrovascular. La lista de verificación es un recurso tecnológico que contribuye a esta toma de decisiones, facilita la práctica profesional y sirve de base para nuevas políticas públicas, ya que aporta beneficios a la salud de los ancianos, además de generar menor tiempo de hospitalización y evitar el empeoramiento de la situación de los ancianos después del accidente cerebrovascular.

Palabras clave: Checklist; Accidente cerebrovascular; Respiración con presión positiva; Ventilación no invasiva.

\section{Introdução}

Sabe-se que com o envelhecimento, o homem torna-se mais vulnerável às doenças e à perda de autonomia, diante da redução natural de suas funções fisiológicas que provoca alterações em sua capacidade funcional e dificulta a realização das tarefas diárias. A fragilidade é uma síndrome que afeta os idosos e representa a combinação de problemas em diferentes áreas do funcionamento humano, como físico, sensorial, psicológico e social; nesse sentido, tem-se que o idoso considerado frágil vai necessitar de cuidados especiais, pois seu risco de adoecimento é maior em comparação aos que não são assim considerados (Gobbens et al., 2010).

Alterações na função respiratória podem acontecer em decorrência da idade; as disfunções da respiração são associadas às elevadas taxas de mortalidade. Estudo indicou que a força do diafragma estava reduzida em indivíduos idosos quando comparados aos mais jovens; tal diminuição relacionada à idade pode predispor os idosos à fadiga do diafragma na presença de condições que prejudicam a função muscular inspiratória ou aumentam a carga ventilatória. Modificações musculares associadas ao envelhecimento comprometem a função dos músculos respiratórios, com diminuição da força diafragmática em idosos (Tolep et al., 1995).

A fragilidade e a disfunção respiratória têm sido identificadas em idosos por meio da realização da espirometria, demonstrando aumento progressivo da rigidez da parede torácica e diminuição do componente elástico dos pulmões, diminuição da massa muscular, força e função, com repercussão nos músculos respiratórios, ensejando possibilidades de impacto no desempenho das atividades de vida diária e na capacidade funcional (Pegorari, Ruas \& Patrizzi, 2013).

Acresce-se que entre os vários acometimentos que podem afetar as pessoas no processo de envelhecer está o Acidente Vascular Cerebral (AVC). Trata-se de uma lesão neurológica vascular que pode ser isquêmica - provocando necrose do tecido cerebral - ou hemorrágica - em que acontece o rompimento de um vaso arterial ou venoso no cérebro, extravasando esse sangue para o espaço intra-parenquimatoso ou para a região subaracnóidea. O indivíduo acometido pode apresentar alterações da força muscular e perda de consciência e sequelas irreversíveis; a maior incidência é em pessoas idosas, em geral com idades iguais ou superiores a 65 anos (Pauli et al., 2020). As manifestações de um AVC comprometem a funcionalidade e limitam a realização das atividades de vida diária, além de restringirem a participação social e comprometerem a qualidade de vida da pessoa acometida (Silva et al., 2020). Ainda, tem-se que os indivíduos com diagnóstico de AVC apresentam comprometimento dos 
movimentos voluntários, espasticidade e perda da atividade seletiva dos músculos responsáveis pelo controle do tronco, que se associa também à função respiratória, já que a expansibilidade e excursão diafragmática dependem da ação de músculos axiais (Pompeu et al., 2011).

Então, além das características inerentes ao envelhecimento, no paciente com a presença deste tipo de alteração neurológica pode ocorrer insuficiência respiratória devido à lesão de estruturas vitais responsáveis pela respiração, excitação e proteção das vias aéreas. Cerca de 12,5\% de todos os pacientes com AVC precisam de ventilação mecânica (VM) e 16,3\% necessitam de traqueostomia (Lahiri et al., 2015).

A VM substitui total ou parcialmente a ventilação espontânea e está indicada na insuficiência respiratória aguda (IRA) ou crônica agudizada. A ventilação não invasiva (VNI) utiliza uma pressão inspiratória para ventilar o paciente por meio de interface externa, geralmente uma máscara facial, através de pressão inspiratória positiva (IPAP) e/ou pressão de suporte (PSV) e uma pressão expiratória positiva (EPAP) ou pressão expiratória final positiva (PEEP), para permitir que as vias aéreas e os alvéolos abertos possam melhorar a oxigenação. No modo de pressão positiva contínua nas vias aéreas (CPAP) é ofertada ao paciente, por interface nasofacial, somente uma pressão expiratória final contínua nas vias aéreas e a ventilação é exclusivamente espontânea (Barbas et al., 2014).

Dentre os benefícios da utilização da VNI, pode-se observar a redução do trabalho respiratório e da frequência respiratória, aumento do volume corrente, melhora da troca gasosa e da dispneia, além da promoção do repouso da musculatura respiratória e o conforto do paciente (Cruz \& Zamora, 2013).

Após um AVC, a reabilitação por meio da fisioterapia comumente prioriza a avaliação e o tratamento de aspectos neurológicos, incluindo a fraqueza muscular do hemicorpo contralateral à lesão cerebral (hemiparesia) e a presença de sinergismo anormal, que dificultam o controle dos movimentos. A recuperação da marcha tem sido o objetivo principal na reabilitação de tais pacientes, sendo sua inserção em serviços de fisioterapia cardiorrespiratória ainda uma prática incomum no Brasil (Ovando et al., 2011).

A fisioterapia respiratória é de grande importância na reabilitação do paciente após o AVC, uma vez que as alterações decorrentes da biomecânica torácica, da imobilidade relativa e da diminuição do reflexo de tosse são comuns nesses indivíduos e parecem ter relação direta com infecções pulmonares e piora do quadro clínico (Demo, 2010). Entretanto, estudo em que foram avaliados 15 pacientes com diagnóstico de AVC, com idades entre 31-88 anos e média de 60,1 anos, mostrou que os acometidos por AVC considerado "crônico" pelo tempo de lesão, podem desenvolver estratégias compensatórias para manter a função respiratória (Pompeu et al., 2011).

Diante do exposto anteriormente, supõe-se que a VNI aplicada precocemente no idoso pós-AVC, pode ser um recurso tecnológico que trará benefícios, visto que favorecerá a melhoria na função pulmonar e propiciará uma melhor qualidade de vida desta população.

Logo, o presente estudo teve como objetivo construir e validar um checklist para indicação de ventilação não invasiva em idosos pós-AVC.

Este instrumento será um facilitador para uma tomada de decisão segura pelos fisioterapeutas, sobre a indicação do uso da VNI em idosos, após esse episódio vascular cerebral.

\section{Metodologia}

Trata-se de um estudo quantitativo do tipo metodológico (Estrela, 2018), realizado em quatro fases, nos meses de outubro a novembro de 2017 (Demo, 2010; Polit \& Beck, 2011).

A amostra foi definida por conveniência, sendo que os participantes desta pesquisa realizaram a validação de conteúdo de um Checklist proposto para indicação de ventilação não invasiva em idosos pós-AVC e compuseram dois grupos com 10 
profissionais cada, denominados GD (grupo de doutores, experts em fisioterapia respiratória) e GF (grupo de fisioterapeutas atuantes em assistência cardiorrespiratória em pessoas idosas), totalizando 20 sujeitos (Medeiros et al., 2015). Para o quantitativo de experts, são sugeridos de seis a vinte sujeitos (Pasquali, 2010). Para o número de participantes nos dois grupos, nossa amostra foi composta por 10 indivíduos por grupo.

O GD foi selecionado de maneira intencional, por meio de pesquisa dos currículos lattes; foi constituído por profissionais da fisioterapia experts na área em estudo, com titulação de doutorado, docentes ou não, que exerciam atividade assistencial ou de ensino, pesquisa e/ou extensão em assistência cardiorrespiratória. O GF foi composto por fisioterapeutas que preencheram ao critério de inclusão de ter no mínimo um ano de experiência na prática assistencial em fisioterapia cardiorrespiratória junto aos idosos, para que tivessem conhecimento sobre a temática, uma vez que a ventilação não invasiva faz parte da atividade assistencial dos fisioterapeutas atuantes no hospital onde ocorreu a coleta de dados. A seleção dos fisioterapeutas ocorreu após aceitação em participarem da pesquisa.

A pesquisa foi realizada no Hospital Universitário Lauro Wanderley (HULW), localizado na cidade de João Pessoa-PB. É um hospital vinculado à Universidade Federal da Paraíba (UFPB) e administrado pela Empresa Brasileira de Serviços Hospitalares (EBSERH), prestando assistência à comunidade nos níveis secundário e terciário.

Este estudo está inserido no projeto intitulado "Políticas, práticas e tecnologias inovadoras para o cuidado na atenção à saúde da pessoa idosa" apreciado pelo Colegiado do Programa de Mestrado Profissional em Gerontologia e submetido à avaliação do Comitê de Ética do Centro de Ciências da Saúde (CCS) e aprovado sob o n ${ }^{\circ}$ 2.190.153, CAAE: 67103917.6.0000.5188. Todos os aspectos da pesquisa envolvendo seres humanos foram considerados segundo a Resolução ${ }^{\circ}$ 466/12 do Conselho Nacional de Saúde (Brasil, 2013).

Para viabilização do estudo, foram propostas as seguintes etapas metodológicas:

1. Realizou-se levantamento de dados na literatura nacional e internacional sobre a temática pertinente ao estudo proposto, sendo gerado um artigo de revisão integrativa. A partir desse estudo, foram detectados os indicadores empíricos para a construção do checklist, sendo elaborada sua primeira versão.

2. O checklist foi apresentado ao GD para a realização da primeira avaliação; solicitou-se que emitissem respostas mediante os itens propostos, que foram divididos em três partes: 1. Apresentação do conteúdo "layout"; 2. Coerência do conteúdo; 3. Sugestões. Foi realizada categorização temática das respostas, agrupando-as com a mesma proposta dos participantes, para formação do parecer; após foi preenchida uma planilha no formato Excel $^{\circledR}$ para favorecer a compilação das respostas ( sim ou não). Os pareceres, após avaliação, deram suporte à segunda versão do checklist que foi gerada e encaminhada para a apreciação do GF.

3. Essa segunda versão do checklist foi entregue, pessoalmente pelos autores, para os componentes do GF do hospital em estudo. A avaliação foi composta por onze questões, sendo: apresentação adequada (layout)?; Está escrito de forma objetiva?; Existe coerência do conteúdo apresentado?; O conteúdo está escrito de forma clara?; A proposta é relevante?; O conteúdo abrange a temática?; Sua aplicação é viável?; Há necessidade de valor numérico (escala/escore) para indicação de VNI?; Pode ser aplicado em curto tempo?; Pode auxiliar o fisioterapeuta na tomada de decisão na indicação de VNI em idosos pós-AVC?; Há necessidade de acréscimo de conteúdo?

As respostas dos fisioterapeutas limitaram-se a "sim" ou "não", sendo ainda disponibilizado um espaço para sugestões. A partir das respostas, foi preenchida a planilha no formato Excel $^{\circledR}$ para favorecer a sua compilação foi realizado o teste de proporções sobre a aplicabilidade (relevância do instrumento, viabilidade de aplicação, abrangência do conteúdo, aplicação em curto tempo, auxílio na tomada de decisão) e a estrutura (apresentação, objetividade, coerência, clareza de conteúdo, acréscimo de conteúdo, necessidade de escala) do checklist. 
4. O material obtido a partir da avaliação GF foi agrupado e analisado à luz da literatura pertinente ao tema investigado e uma terceira versão do checklist foi elaborada. As demais fases de validação de conteúdo serão realizadas a posteriori (Medeiros et al., 2015).

5. Os dados foram analisados estatisticamente, por meio de análise descritiva e de testes inferenciais: Testes de proporções foram realizados para verificar a diferença nas opiniões dos avaliadores sobre aplicabilidade (relevância do instrumento, viabilidade de aplicação, abrangência do conteúdo, aplicação em curto tempo, auxílio na tomada de decisão) e estruturação (apresentação, objetividade, coerência, clareza de conteúdo, acréscimo de conteúdo, necessidade de escala) do checklist; utilizou-se o teste Chi-quadrado de Pearson, a fim de comparar a avaliação de fisioterapeutas especialistas e fisioterapeutas assistenciais. Definiu-se nível de significância menor que 0,05.

\section{Resultados e Discussão}

As opiniões dos participantes sobre a aplicabilidade e a estrutura do checklist estão apresentadas nas Tabelas 1 e 2, apresentadas na sequência.

Quadro 1: Distribuição dos fisioterapeutas especialistas e assistenciais segundo suas opiniões sobre a aplicabilidade do checklist para indicação de ventilação não invasiva em idosos pós Acidente Vascular Cerebral. João Pessoa, Paraíba, 2017. (n=20).

\begin{tabular}{|l|c|c|c|c|c|c|c|}
\hline \multirow{2}{*}{ Variáveis } & \multicolumn{2}{|c|}{ Sim } & \multicolumn{2}{c|}{ Não } & \multicolumn{2}{c|}{ Não relata } & \multirow{2}{*}{ p-valor } \\
\cline { 2 - 7 } & $\mathbf{N}$ & $\mathbf{\%}$ & $\mathbf{N}$ & $\mathbf{\%}$ & $\mathbf{N}$ & $\mathbf{\%}$ & \\
\hline Relevância do instrumento & 16 & 80,0 & 0 & 0,0 & 4 & 20,0 & $0,007 * *$ \\
\hline Viabilidade de aplicação & 20 & 100 & 0 & 0,0 & 0 & 0,0 & 1,0 \\
\hline Abrangência de conteúdo & 10 & 50,0 & $10^{*}$ & $50,0 *$ & 0 & 0,0 & 1,0 \\
\hline Aplicação em curto tempo & 19 & 95,0 & 0 & 0,0 & 1 & 5,0 & $0,0001^{* *}$ \\
\hline Auxílio na tomada de decisão & 20 & 100 & 0 & 0,0 & 0 & 0,0 & 1,0 \\
\hline
\end{tabular}

Teste para diferença de Proporções; significância $\mathrm{p}<0,05^{* *}$; Fonte: Autores.

De modo geral, observou-se que a aplicabilidade do instrumento foi avaliada positivamente, tendo em vista a opinião unânime dos avaliadores em relação à sua viabilidade de aplicação e ao auxílio na tomada de decisão sobre a indicação da ventilação mecânica $(n=20 ; 100 \%)$. Sua relevância na área e na prática clínica também foi confirmada pela maioria da amostra, com proporção significante ( $\mathrm{p}=0,007)$, bem como a possibilidade de aplicação em curto tempo (p=0,0001) (Quadro 1).

Sobre a abrangência de conteúdo, $50 \%(\mathrm{n}=10)$ relataram que o checklist é abrangente e os demais afirmaram que é necessário acréscimo de conteúdo para torná-lo mais completo, demonstrando que houve divergência entre os examinadores quanto a essa variável (Quadro 1).

A Tabela 1 a seguir expõe dados sobre a opinião dos fisioterapeutas sobre a estrutura do instrumento. Eles foram unânimes em afirmar que ele possui boa apresentação, é objetivo, coerente e claro em seu conteúdo (n=20; 100\%). 
Tabela 1: Distribuição dos fisioterapeutas especialistas e assistenciais segundo suas opiniões sobre a estrutura do checklist para indicação de ventilação não invasiva em idosos pós Acidente Vascular Cerebral. João Pessoa, Paraíba, 2017. (n=20).

\begin{tabular}{lccccccc}
\hline \multirow{2}{*}{ Variáveis } & \multicolumn{2}{c}{ Sim } & \multicolumn{2}{c}{ Não } & \multicolumn{2}{c}{ Não relata } & \multirow{2}{*}{ p-valor } \\
\cline { 2 - 6 } & $\mathbf{N}$ & $\mathbf{\%}$ & $\mathbf{N}$ & $\mathbf{\%}$ & $\mathbf{N}$ & $\mathbf{\%}$ & \\
\hline Apresentação & 20 & 100 & 0 & 0,0 & 0 & 0,0 & 1,0 \\
Objetividade & 20 & 100 & 0 & 0,0 & 0 & 0,0 & 1,0 \\
Coerência & 20 & 100 & 0 & 0,0 & 0 & 0,0 & 1,0 \\
Clareza de conteúdo & 20 & 100 & 0 & 0,0 & 0 & 0,0 & 1,0 \\
Acréscimo de conteúdo & 14 & 70,0 & 6 & 30,0 & 0 & 0,0 & $0,044^{*}$ \\
Necessidade de escala & 8 & 40,0 & 5 & 25,0 & 7 & 35,5 & 0,705 \\
\hline
\end{tabular}

Teste para diferença de Proporções; significância p<0,05*; Fonte: Autores.

No que diz respeito à necessidade de acréscimo de conteúdo, 14 participantes (70\%) afirmaram haver necessidade, sendo que essa frequência sobressaiu em relação à de não haver necessidade $(n=6 ; 30 \%)$ de forma proporcionalmente significante $(\mathrm{p}=0,044)$.

Para as variáveis em apresentaram diferença de opinião, realizou-se associação a fim de verificar separadamente a concordância das opiniões de fisioterapeutas especialistas e fisioterapeutas assistenciais sobre cada uma delas, como demonstra a Tabela 2.

Tabela 2: Distribuição dos fisioterapeutas especialistas e assistenciais segundo a relação entre suas opiniões sobre a aplicabilidade e a estrutura do checklist para indicação de ventilação não invasiva em idosos pós Acidente Vascular Cerebral. João Pessoa, Paraíba, 2017. $(n=20)$.

\begin{tabular}{|c|c|c|c|c|c|c|c|c|c|c|c|c|c|}
\hline \multirow{3}{*}{ Variáveis } & \multicolumn{6}{|c|}{ Fisioterapeutas especialistas } & \multicolumn{6}{|c|}{ Fisioterapeutas assistenciais } & \multirow{3}{*}{ p-valor } \\
\hline & \multicolumn{2}{|c|}{ Sim } & \multicolumn{2}{|c|}{ Não } & \multicolumn{2}{|c|}{$\begin{array}{c}\text { Não } \\
\text { relata }\end{array}$} & \multicolumn{2}{|c|}{ Sim } & \multicolumn{2}{|c|}{ Não } & \multicolumn{2}{|c|}{$\begin{array}{c}\text { Não } \\
\text { relata }\end{array}$} & \\
\hline & $\mathbf{N}$ & $\%$ & $\mathbf{N}$ & $\%$ & $\mathbf{N}$ & $\%$ & $\mathbf{N}$ & $\%$ & $\mathbf{N}$ & $\%$ & $\mathbf{N}$ & $\%$ & \\
\hline Relevância do instrumento & 6 & 60,0 & 0 & 0,0 & 4 & 40,0 & 10 & 100 & 0 & 0,0 & 0 & 0,0 & 0,073 \\
\hline Aplicação em curto tempo & 9 & 90,0 & 0 & 0,0 & 1 & 10,0 & 10 & 100 & 0 & 0,0 & 0 & 0,0 & 0,500 \\
\hline Necessidade de escala & 3 & 30,0 & 0 & 0,0 & 7 & 70,0 & 5 & 50,0 & 5 & 50,0 & 0 & 0,0 & $0,0001 *$ \\
\hline Abrangência de conteúdo & 0 & 0,0 & 10 & 100 & 0 & 0,0 & 10 & 100 & 0 & 0,0 & 0 & 0,0 & $0,0001 *$ \\
\hline Acréscimo de conteúdo & 7 & 70,0 & 3 & 30,0 & 0 & 0,0 & 7 & 70,0 & 3 & 30,0 & 0 & 0,0 & 0,686 \\
\hline
\end{tabular}

Teste de Chi-Quadrado de Pearson; significância p<0,05* Fonte: Autores.

Observou-se que a maioria dos doutores $(n=6 ; 60 \%)$ considerou o instrumento relevante, sendo que os demais não pontuaram essa variável. Todos os avaliadores reconheceram a relevância do instrumento em sua prática clínica. Da mesma forma ocorreu com a variável "aplicação em curto tempo", em que grande maioria dos doutores (n=9; 90\%) e todos os fisioterapeutas assistenciais $(10 ; 100 \%)$ concordaram que o checklist é aplicável em pouco tempo. Dessa forma, independente do grupo de avaliadores, a relevância do instrumento foi confirmada ( $\mathrm{p}=0,073)$, bem como a sua rápida aplicação ( $\mathrm{p}=0,500)$ (Tabela 2).

Em relação à necessidade de escala calculável, observou-se maior frequência no grupo dos fisioterapeutas assistenciais $(\mathrm{n}=5 ; 50 \%)$, que são atuantes na área. A maioria dos que são doutores não relatou sobre esse aspecto $(\mathrm{n}=7 ; 70 \%)$. Essa diferença de opiniões foi estatisticamente significante $(\mathrm{p}=0,0001)$, ou seja, a necessidade de escala vai ser importante, dependendo do grupo de avaliadores.

Os doutores não concordaram com a abrangência do instrumento em relação ao conteúdo relacionado à indicação da ventilação mecânica $(n=10 ; 100 \%)$. Já os fisioterapeutas assistenciais concordaram de forma unânime. Dessa forma, percebeu-se que em relação a essa variável, houve divergência, sendo ela estatisticamente significante (p=0,0001). 
Ambos os grupos $(\mathrm{n}=7 ; 70 \%)$ concordaram $(\mathrm{p}=0,686)$ que seriam necessários acréscimos de conteúdo no checklist, para que ele se tornasse mais eficaz. Os avaliadores deram sugestões como: incluir data de admissão e alta hospitalar, tempo de internação, peso corpóreo, intubação prévia, avaliação neurológica, déficit motor, avaliação hemodinâmica, entre outras.

As sugestões do GD foram: data de admissão e da alta hospitalar, peso corpóreo predito, inclusão do reflexo de tosse e da deglutição; acréscimo, na avaliação inicial, dos itens agitação e cooperação, exclusão de hipertensão e manutenção de hipertensão não controlada, alteração de avaliação da qualidade do sono para avaliação do sono, informação se houve intubação prévia ou não, presença de sinais de infecção respiratória, exclusão ou reformulação do item critérios para intubação imediata, Frequência respiratória (FR), indicação dos padrões de bradipneia, mudança saturação de oxigênio no sangue arterial $\left(\mathrm{SatO}_{2}\right)$ para saturação periférica de oxigênio no sangue arterial $\left(\mathrm{SpO}_{2}\right)$, acréscimo de presença de sonda nasogástrica ou enteral, presença de engasgo ao se alimentar, presença de secreção, tabagista sim ou não, presença de ritmo respiratório patológico, tipo de AVC, acréscimo de tempo de acometimento do AVC, revisão dos itens 15 e 16 sobre hipertensão, revisão do valor da Frequência cardíaca (FC) na literatura, ou seja, FC< 140 batimentos por minuto (bpm), item 15 normotenso ou hipertensão controlada dividir em dois aspectos e item 16 atualizar os valores de pressão arterial (PA). Estas sugestões foram consideradas mediante respaldo na literatura e foram acrescidas ao checklist.

O GF também realizou várias sugestões pertinentes, como: se houve necessidade de uso de ventilação mecânica invasiva antes da instalação da VNI, separar as contraindicações, calcular o peso predito; calcular a pressão parcial de oxigênio no sangue arterial $\left(\mathrm{PaO}_{2}\right)$ de acordo com a idade, acrescentar como contraindicação claustrofobia, colocar que se o paciente não passar pela avaliação inicial não deve passar para as demais fases do checklist.

Assim, na identificação do paciente algumas informações foram acrescentadas de acordo com as sugestões, como: data de admissão, peso predito de acordo com a altura e o sexo, tipo de AVC, tempo de acometimento do AVC, presença de outras doenças respiratórias e se já fez uso de ventilação mecânica invasiva (VMI). Todas as sugestões tiveram relação com informações que auxiliaram na elaboração do checklist, seja para situar o fisioterapeuta no caso clínico, seja para oferecer informações que auxiliarão no decorrer da utilização do checklist.

$\mathrm{Na}$ avaliação da ventilação e do sono, não houve sugestões de acréscimo de conteúdo. As sugestões que não foram pertinentes no GD seriam melhor analisadas utilizando-se de escore em cada nível de avaliação ou de forma geral, para que a indicação da VNI se torne mais objetiva. No GF também houve sugestão de atribuição de escores para a quantificação de uso da VNI, além de ser solicitada a inclusão do mini exame de estado mental (MEEM) como critério de inclusão.

A partir do exposto anteriormente o checklist elaborado apresenta-se a seguir (Figura 1). 
Figura 1. Checklist.

\begin{tabular}{|c|c|c|c|}
\hline \multicolumn{4}{|c|}{ IDENTIFICAÇÃO DO PACIENTE } \\
\hline \multicolumn{4}{|c|}{ PRONT.: } \\
\hline \multicolumn{4}{|c|}{ DATA DE ADMISSÃO: } \\
\hline \multicolumn{4}{|c|}{ ALTURA:__ PESO ATUAL__ } \\
\hline \multicolumn{4}{|c|}{$\begin{array}{l}\text { PESO PREDITO __ Kg (altura em } \mathrm{cm}-152,4 \text { x } 0,91+50) \text { homens (altura em } \mathrm{cm}-152,4 \times 0,91+45) \\
\text { mulheres }\end{array}$} \\
\hline \multicolumn{4}{|c|}{ 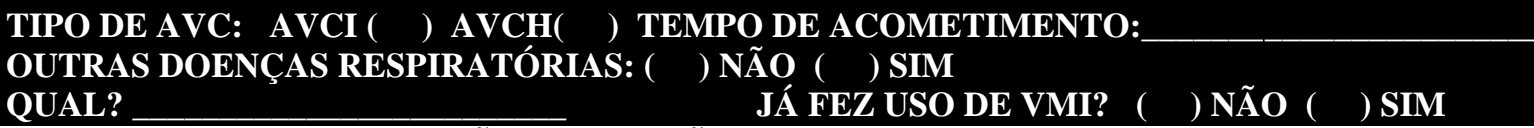 } \\
\hline \multicolumn{4}{|c|}{$\begin{array}{llll}\text { LEGENDA : } & \text { S- SIM } & \text { N- NÃO } & \text { NA - NÃO SE APLICA } \\
\end{array}$} \\
\hline \multicolumn{4}{|c|}{ CONTRAINDICAÇÕES DE VNI } \\
\hline 1.AGITAÇÃO & S ( & $\mathrm{N}($ & NA ( \\
\hline 2. CONSCIENTE (ESCALA DE GLASGOW <12)* & S ( & $\mathrm{N}($ & NA ( \\
\hline 1. OBSTRUÇÃO MECÂNICA DA VIA AÉREA ALTA & S ( & $\mathrm{N}($ & NA ( \\
\hline 2. DISTENSÃO ABDOMINAL & $\mathrm{S}($ & $\mathrm{N}($ & NA ( \\
\hline 3. PNEUMOTÓRAX NÃO DRENADO & S ( & $\mathrm{N}($ & NA ( \\
\hline 4. VÔMITOS & S ( & $\mathrm{N}($ & NA ( \\
\hline 5. SANGRAMENTO DIGESTIVO ALTO & S ( & $\mathrm{N}($ & NA ( \\
\hline 6. SÍNDROME CORONARIANA AGUDA & S ( & $\mathrm{N}($ & NA ( \\
\hline $\begin{array}{l}\text { 7. CIRURGIA ESOFÁGICA, FACE E/OU DE VIA AÉREA } \\
\text { (RECENTE) }\end{array}$ & S ( & $\mathrm{N}($ & NA ( \\
\hline 8. ARRITMIAS CARDÍACAS COMPLEXAS & S ( & $\mathrm{N}($ & NA ( \\
\hline 9.TRAUMA DE FACE & S ( & $\mathrm{N}($ & NA ( \\
\hline 10. CLAUSTROFOBIA & S ( & $\mathrm{N}($ & NA ( \\
\hline
\end{tabular}

- ESCALA DE GLASGOW PERMITE MEDIR/AVALIAR O NÍVEL DE CONSCIÊNCIA. CASO ALGUM DOS ITENS ACIMA TENHA A RESPONTA "SIM", A VENTILAÇÃO NÃO INVASIVA ESTÁ CONTRAINDICADA!

\section{AVALIAÇÃO INICIAL}

\begin{tabular}{|c|c|c|c|}
\hline 11. CONSCIENTE & S ( & $\mathrm{N}($ & NA ( \\
\hline 12. TOSSE EFICAZ & S ( & $\mathrm{N}($ & NA ( \\
\hline 13. ENGASGO AO SE ALIMENTAR & S ( & $\mathrm{N}($ & NA ( \\
\hline 14. SECREÇÃO EM VIAS ÁREAS SUPERIORES OU INFERIORES & S ( & $\mathrm{N}($ & NA ( \\
\hline 15. INFECÇÃO RESPIRATÓRIA & S ( & $\mathrm{N}($ & NA ( \\
\hline $\begin{array}{l}\text { 16. ALIMENTAÇÃO POR VIA ALTERNATIVA NASAL OU ORAL } \\
\text { (SONDA) }\end{array}$ & $\mathrm{S}(\quad)$ & $\mathrm{N}($ & NA ( \\
\hline
\end{tabular}

AVALIAÇÃO HEMODINÂMICA

\begin{tabular}{|c|c|c|c|}
\hline 17. FC > $60 \mathrm{bpm}$ & $S(\quad)$ & $\mathrm{N}(\quad)$ & NA ( \\
\hline 18. FC $<140 \mathrm{bpm}$ & S ( & $\mathrm{N}(\quad)$ & NA ( \\
\hline 19. NORMOTENSO & S ( & $\mathrm{N}($ & NA ( \\
\hline 20. HIPERTENSÃO (PAS $\geq 140 \mathrm{mmHg}$ E/OU PAD $\geq 90 \mathrm{mmHg}$ ) & S ( ) & $\mathrm{N}(\quad)$ & NA ( \\
\hline \multicolumn{4}{|l|}{ AVALIAÇÃO DA RESPIRAÇÃO } \\
\hline 21. FR > $35 \mathrm{rpm}$ & $\mathrm{S}(\quad)$ & $\mathrm{N}($ & NA ( \\
\hline 22. FR $<12 \mathrm{rpm}$ & S ( & N ( & NA ( \\
\hline 23. PADRÃO RESPIRATÓRIO PATOLÓGICO & S ( & $\mathrm{N}($ & NA ( \\
\hline $\begin{array}{l}\text { 24. USO DE MUSCULATURA ACESSÓRIA COM AUMENTO DO } \\
\text { TRABALHO VENTILATÓRIO }\end{array}$ & S ( & N ( & NA ( \\
\hline \multicolumn{4}{|l|}{ AVALIAÇÃ̃ DA OXIGENAÇÃO } \\
\hline 25. NECESSIDADE DE SUPORTE DE OXIGÊENIO COM $\mathrm{FiO}_{2}>50 \%$ & S ( & $\mathrm{N}($ & NA ( \\
\hline 26. $\mathrm{SpO}_{2}<90 \%$ & S ( & $\mathrm{N}($ & NA ( \\
\hline $\begin{array}{l}\text { 27. } \mathrm{PaO}_{2}<60 \mathrm{mmHg} \\
\mathrm{PaO}_{2}=109-0,45 \times \text { idade (AR AMBIENTE) }\end{array}$ & $\mathrm{S}(\quad)$ & $\mathrm{N}(\quad)$ & NA ( \\
\hline 28. $\mathrm{PaO}_{2} / \mathrm{FiO}_{2}<300 \mathrm{mmHg}$ & $\mathrm{S}(\quad)$ & $\mathrm{N}($ & NA ( \\
\hline \multicolumn{4}{|l|}{ AVALIAÇÃO DA VENTILAÇÃO } \\
\hline 29. VC REDUZIDO (5 a $8 \mathrm{ml} / \mathrm{Kg}$ peso) & $\mathrm{S}($ & $\mathrm{N}($ & NA ( \\
\hline 30. VM INADEQUADO (VC x FR) & S ( & $\mathrm{N}($ & NA ( \\
\hline 31. pH GASOMÉTRICO < 7,35 & $S(\quad)$ & $\mathrm{N}($ & NA ( \\
\hline 32. $\mathrm{PaCO}_{2}>45 \mathrm{mmHg}$ & $S(\quad)$ & $\mathrm{N}($ & NA ( \\
\hline
\end{tabular}




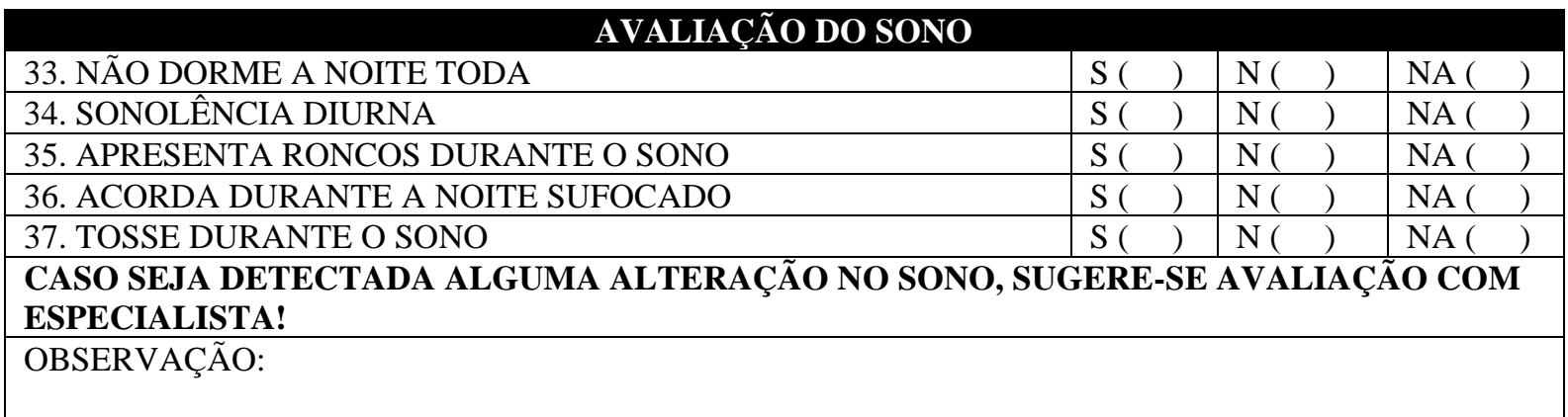

Fonte: Autores.

\section{Discussão}

Constatou-se no presente estudo, que nos pareceres emitidos por fisioterapeutas especialistas e assistenciais houve convergência de informações na maior parte dos itens avaliados para serem inseridos ou não no checklist, o que incluiu a aplicabilidade e a estrutura. Apenas na abrangência de conteúdo houve divergência, o que contribuiu de forma a repensar sobre o instrumento, favorecendo a melhoria da sua aplicabilidade, para assim construir sua $3^{\mathrm{a}}$ versão, produto desta pesquisa.

Ressalta-se que essa divergência provavelmente esteja relacionada ao fato dos fisioterapeutas assistenciais atuarem na área cardiorrespiratória e ter uma visão apoiada na prática, destacando assim, a prática baseada em evidência que tanto se discute atualmente. Neste contexto, o GD composto por fisioterapeutas doutores atuantes em atividades acadêmicas relacionadas à temática, talvez tenham se preocupado com o método e não consideraram variáveis relacionadas ao contexto hospitalar que o GF, composto por fisioterapeutas assistenciais consideraram.

A imobilidade, descondicionamento físico e fraqueza muscular são problemas frequentemente encontrados em pacientes sob ventilação mecânica. A imobilidade acontece com maior importância nos músculos respiratórios, pelo fato do ventilador mecânico assumir uma proporção maior do trabalho respiratório, reduzindo o trabalho exercido pela ventilação espontânea. Isso resulta na ausência completa ou parcial da ativação neural e da mecânica muscular, reduzindo, assim, a capacidade que o diafragma tem de gerar força (Dantas et al., 2012). Com a imobilidade, descondicionamento físico e fraqueza muscular, o paciente poderá apresentar alterações na frequência respiratória, no padrão respiratório e na utilização da musculatura acessória. O que reforça a importância da utilização da ventilação não invasiva precoce.

O paciente com AVC pode ter doença respiratória associada, neste caso, quando há a necessidade de suporte ventilatório, deve-se tentar inicialmente a instituição de Ventilação Não Invasiva (VNI). Essa é considerada o tratamento de excelência e está baseada em ampla literatura a qual ela se relaciona com menor taxa de intubação traqueal, menor risco de infecções, menor tempo de internação em Unidade de Terapia Intensiva (UTI) e, consequentemente, menor mortalidade (Pincelli et al., 2011) Também foi importante predizer o peso do paciente para estabelecer estratégias terapêuticas ajustadas às suas necessidades individuais, inclusive para a determinação do volume corrente (VC) necessário e adequado ao paciente sob VM (Viana et al., 2014).

No item avaliação inicial, foi acatada a sugestão quanto a discriminação das contraindicações de VNI. Os avaliadores recomendaram que o paciente não tenha qualquer das contraindicações de VNI para que seja instituída e sugeriram acrescentar agitação e claustrofobia. A causa para o insucesso da VNI é multifatorial e a agitação é uma delas (Hess, 2013).

A intolerância à VNI é uma das razões para o insucesso da terapia. A VNI pode falhar em 5 a $40 \%$ dos casos, associandose a quatro vezes mais chance de mortalidade hospitalar. Os problemas associados à interface são os efeitos adversos mais frequentes, representando 50 a 100\% de todas as complicações, como, por exemplo, vazamentos excessivos, sensação desconfortável da pressão de ar na face, claustrofobia, reinalação de dióxido de carbono, lesões de pele, dor facial e ressecamento oronasal (Pontes et al., 2017). 
Também foram acrescidos na avaliação inicial os itens tosse eficaz, engasgo ao se alimentar, secreção em vias aéreas superiores ou inferiores, infecção respiratória, alimentação por via alternativa nasal ou oral (sonda). A tosse é o principal mecanismo para manter as vias aéreas limpas e é eficaz na redução do risco de pneumonia por aspiração. Na ventilação não invasiva, são considerados fatores de risco para falência respiratória tosse ineficaz e secreção (Barbas et al., 2014).

O distúrbio na função da deglutição pode resultar em regurgitação nasal ou aspiração de alimentos. Assim, a disfagia geralmente é associada com tosse após a deglutição, dificuldade respiratória, infecção pulmonar, comprometendo tanto a parte respiratória do indivíduo como a sua nutrição. Outra pesquisa apresentou uma incidência de disfagia de $30 \%$ a $50 \%$ entre pacientes vítimas do AVC, a qual é considerada um dos fatores de risco para a pneumonia aspirativa e uma das complicações mais frequentes relacionadas à essa alteração neurológica. Em pacientes com AVC e disfágicos, o risco para desenvolver pneumonia aspirativa é 3,17 vezes maior do que no paciente acamado sem disfagia e 11,5 vezes maior para o risco de aspiração. Acredita-se que isso se deva à aspiração, algumas vezes silenciosa, relacionada à disfagia resultante da lesão encefálica (Paixão, Silva \& Camerini, 2010).

Foi acrescido na avaliação hemodinâmica o ajuste do valor da FC de 100bpm para 140bpm como limite máximo e os termos normotenso ou hipertensão controlada, hipertensão ou hipertensão não controlada; a alteração foi de acordo com a classificação do Sétimo Relatório do Comitê Nacional Conjunto sobre Prevenção, Detecção, Avaliação e Tratamento da pressão arterial (PA) Elevada (Chobanian et al., 2003). Hipertensão arterial (HA) é condição clínica multifatorial caracterizada por elevação sustentada dos níveis pressóricos $\geq 120$ a 140 mmHg e/ou 80 ou 90 mmHg. Comumente associa-se aos distúrbios metabólicos, alterações funcionais e/ou estruturais de órgãos-alvo, sendo intensificada pela presença de outros fatores de risco (FR), como dislipidemia, obesidade abdominal, intolerância à glicose e diabetes mellitus (DM). Mantém associação independente com eventos como morte súbita, acidente vascular cerebral (AVC), infarto agudo do miocárdio (IAM), insuficiência cardíaca (IC), doença arterial periférica (DAP) e doença renal crônica (DRC), fatal e não fatal (Malachias et al., 2016). A avaliação da FC < 140 bpm é um parâmetro utilizado para avaliar a intolerância ao esforço físico e mecanismos de falência respiratória (Goldwasser et al., 2007).

No item avaliação da respiração, foi sugerido inserir o valor de bradipneia, a fórmula para o cálculo de $\mathrm{PaO}_{2}$ de acordo com a idade e o padrão respiratório patológico. A bradipneia corresponde a frequência respiratória abaixo de 10 rpm (respirações por minuto), geralmente está relacionada à depressão do sistema nervoso central (Pryor \& Webber, 2002). $\mathrm{O}$ cálculo da $\mathrm{PaO}{ }_{2}$ ideal leva em consideração a idade, paciente em posição supina e sua fórmula é $\mathrm{PaO}_{2}=109-(0,43 \mathrm{x}$ idade). Os idosos apresentam uma $\mathrm{PaO}_{2}$ normalmente mais baixa (Guedes et al., 2013). Cargas respiratórias internas, induzidas por doenças do sistema respiratório, também podem modificar o padrão respiratório (Machado, 2008).

Logo, após as sugestões dos fisioterapeutas doutores e dos fisioterapeutas que atuavam em assistência cardiorrespiratória, foi elaborada a versão final do checklist, para que, posteriormente, ele possa ser aplicado nos idosos pósAVC, o que possibilitará verificar sua aplicabilidade e inserção de escore, para que os fisioterapeutas indiquem a VNI embasados em um instrumento norteador para sua conduta terapêutica.

O checklist é um recurso tecnológico que contribui com a tomada de decisão para indicação de ventilação não invasiva em idosos pós-acidente vascular cerebral. Facilita a prática profissional e serve como base para novas políticas públicas, quando aplicado precocemente, pode ser capaz de trazer benefícios à saúde da pessoa idosa, visto que poderá favorecer a melhoria na função pulmonar, melhor qualidade de vida, além de gerar menor tempo de internação e evitar a piora no quadro do idoso pós acidente vascular cerebral.

O checklist produzido neste estudo precisa passar pela validação clínica, para que seja avaliada sua aplicabilidade nos serviços de saúde. É importante que o fisioterapeuta possua conhecimento acerca da utilização da ventilação não invasiva, pois a falta de conhecimento pode ser um fator limitante na tomada de decisão, assim como a disponibilidade do aparelho de 
ventilação não invasiva nos hospitais, pois de nada servirá instituir o checklist se não houver a possibilidade da utilização deste recurso terapêutico.

Como limitação do estudo, é possível apontar a necessidade de coletar junto a mais fisioterapeutas atuantes na assistência cardiorrespiratória de pessoas idosas, já que serão esses os profissionais que utilizarão o checklist. Ressalta-se que a amostra foi definida por conveniência e não de forma aleatória. E há necessidade de mais pesquisas que envolvam esse tipo de método para promover a tomada de decisão nesse contexto de atuação.

\section{Conclusão}

Os resultados possibilitaram construir e validar um checklist de 37 itens que orienta quanto a indicação de ventilação não invasiva em idosos pós-acidente vascular cerebral e que facilita a tomada de decisão dos fisioterapeutas. As sugestões pertinentes levadas em consideração na sua elaboração foram consideradas mediante respaldo da literatura existente e situam o fisioterapeuta na condução do caso clínico, oferecendo informações relevantes que servirão como base no decorrer da utilização do instrumento (outras doenças respiratórias, cálculo do peso predito e se fez uso de VMI, data de admissão e tempo de acometimento do acidente vascular cerebral, entre outras).

Este instrumento se aplicado precocemente, pode ser um recurso tecnológico, capaz de trazer benefícios a saúde da pessoa idosa, visto que favorecerá melhoria na função pulmonar e propiciará uma melhor qualidade de vida. Espera-se que este checklist traga benefícios para a área da gerontologia, e que por meio das condutas e práticas abordadas, seja possível promover um envelhecimento saudável, evitando a piora no quadro do idoso e internações prolongadas, bem como, acredita-se que o instrumento produzido facilite a prática profissional e a educação em saúde entre os profissionais da saúde.

Trabalhos futuros são importantes, para avaliação do checklist por fisioterapeutas atuantes na assistência cardiorrespiratória de pessoas idosas, já que serão esses os profissionais que utilizarão o checklist. Além, da perspectiva de criação de score, para conduzir a tomada de decisão precoce da indicação da ventilação não invasiva em idosos pós acidente vascular cerebral.

\section{Referências}

Barbas, C. S., Isola, A. M., Farias, A. M., Cavalcanti, A. B., Gama, A. M., Duarte, A. C., Vianna, A., Serpa Neto, A., Bravim, B. A,, Pinheiro, B. V., Mazza, B. F., Carvalho, C. R., Toufen Jr, C., David, C. M., Taniguchi, C., Mazza, D. D., Dragosavac, D., Toledo, D. O., Costa, E. L., Caser, E. B., Silva, E., Amorim, F. F., Saddy, F., Galas, F. R., Silva, G. S., Matos, G. F., Emmerich, J. C., Valiatti, J. L., Teles, J. M., Victorino, J. A., Ferreira, J. C., Prodomo, L. P., Hajjar, L. A., Martins, L. C., Malbouisson, L. M., Vargas, M. A., Reis, M. A., Amato, M. B., Holanda, M. A., Park, M., Jacomelli, M., Tavares, M., Damasceno, M. C., Assunção, M. S., Damasceno, M. P., Youssef, N. C., Teixeira, P. J., Caruso, P., Duarte, P. A., Messeder, O., Eid, R. C., Rodrigues, R. G., Jesus, R. F., Kairalla, R. A., Justino, S., Nemer, S. N., Romero, S. B, \& Amado, V. M. (2014). Recomendações brasileiras de ventilação mecânica. Rev. Bras. Ter Intensiva; 26(2):89121 .

Brasil (2013). Conselho Nacional de Saúde. Resolução n 466, 2012. Diretrizes e Normas regulamentadoras de pesquisa envolvendo seres humanos.

Chobanian' A. V., Bakris, G. L., Black, H. R., Cushman, W, C., Green, L. A, .Joseph Jr, L. I., Jones, D. W., Materson, B. J., Suzanne Oparil, S., Jackson T Wright Jr, J. T. W., \& Roccella, E. J. (2003). Seventh report of the joint national committee on prevention, detection, evaluation, and treatment of high blood pressure. Hypertension;42(6):1206-52.

Cruz M. R. \& Zamora V. E. C. (2013). Ventilação mecânica não invasiva. Rev.HUPE; 12(3):92-101.

Dantas, C. M., Silva, P. F. S., Siqueira, F. H. T., Pinto, R. M. F., Matias, S., Maciel, C., Oliveira, M. C. O., Albuquerque, C. G., Andrade, F. M. D., Ramos, F. F., \& França, E. E. T. (2012) Influência da mobilização precoce na força muscular periférica e respiratória em pacientes críticos. Rev. bras. ter. intensiva; 24(2):173-178.

Demo P. (2010). Metodologia científica em ciências sociais. Atlas.

Estrela, C. (2018). Metodologia Científica: Ciência, Ensino, Pesquisa. Artes Médicas.

Gobbens, R. J. J., Luijkx, K. G., Wijnen-Sponselee, M. T., \& Schols, J. M. G. A.(2010). In Search of an Integral Conceptual Definition of Frailty: Opinions of Experts. J Am Med Dir Assoc.; 11(5): 338-343. 
Goldwasser, R., Farias, A., Freitas, E. E., Saddy, F., Amado, V., \& Okamoto, V. (2007). Desmame e interrupção da ventilação mecânica. J. bras. pneumol.; 33(2):128-136.

Guedes, L. P. C. M., Delfino, F. C., Farias, F. P., Melo, G. F., \& Carvalho G. A. (2013). Adequação dos parâmetros de oxigenação em idosos submetidos à ventilação mecânica. Einstein; 11(4):467-471.

Hess, D. R. (2013). Noninvasive Ventilation for Acute Respiratory Failure. Respiratory Care, 58 (6), 950-972..

Lahiri, S., Navi, B. B., Mayer, S. A., Rosengart, A., Merkler, A. E., Claassen, J., \& Kamel, H. (2015) Hospital readmission rates among mechanically ventilated patients with stroke. Stroke; 46(10):2969-71.

Machado, M. G. R.. (2008). Bases de fisioterapia respiratória: terapia intensiva e reabilitação. Guanabara Koogan.

Malachias, M. V.B., Póvoa, R. M. S., Nogueira, A. R., Souza, D., Costa, L. S., \& Magalhães, M. E. (2016). $7^{\circ}$ Diretriz Brasileira de hipertensão Arterial: Capítulo 3. Avaliação Clínica e Complementar. Arq. Bras. Cardiol.; 107(3):14-17.

Medeiros, R. K. S., Ferreira Jr, M. A., Pinto, D. P. S. R., Vitor, A. F., Santos, V. E. P., \& Barichello, E. (2015). Modelo de validação de conteúdo de Pasquali nas pesquisas em Enfermagem. Rev. de Enferm Ref. 4(1):127-135.

Ovando, A. C., Michaelsen, S. M., Carvalho, T., \& Herber. V. (2011). Avaliação da Aptidão Cardiopulmonar em Indivíduos com Hemiparesia após-acidente Vascular. Arq. Bras. Cardiol; 96(2):140-147.

Paixão, C. T, Silva, L. D., \& Camerini, F. G. (2010). Perfil da disfagia após um acidente vascular cerebral: uma revisão integrativa. Rev. Rene.11(1):181-190.

Pasquali,, L.(2010) Instrumentação psicológica: Fundamentos e práticas. Artmed.

Pauli, E., Leite, M. T., Bornholdt, L., Hildebrandt, L. M., Kinalski, S. S., \& Beuter, M. (2020). O viver de idosos após o acidente vascular cerebral. Rev. Enferm. UFSM, Santa Maria,10(1): 1-22.

Pegorari, M.S., Ruas, G., \& Patrizzi L. J. (2013). Relationship between frailty and respiratory function in the community-dwelling elderly. Braz. J. Phys. Ther; 17(1):09-16.

Pincelli, M. P., Grumann, A. C. B., Fernandes, C., Cavalheiro, A. G. C., Haussen, D. A. P., \& Maia, I. S. (2011). Características de pacientes com DPOC internados em UTI de um hospital de referência para doenças respiratórias no Brasil. J. bras. pneumol; 37(2):217-222.

Polít,. D. F., \& Beck, C. T. (2011). Fundamentos de pesquisa em enfermagem: avaliação de evidências para as práticas da enfermagem.7a ed. Porto Alegre (RS): Artmed.

Pompeu, S. M. A. A., Pompeu, J. E., Rosa, M., \& Silva, M. R. da. (2011). Correlação entre função motora, equilíbrio e força respiratória pós Acidente Vascular Cerebral. Revista Neurociências:19(4), 614-620.

Pontes, S. M. M., Melo, L. H. P., Maia, N. P. S., Nogueira, A. N. C., Vasconcelos, T. B., Pereira, E. D, B. P., Bastos, V. P. D., \& Holanda, M, A. (2017). Influência do modo ventilatório nos efeitos adversos agudos e na termografia da face após ventilação não invasiva. J. bras. Pneumol, 43(2):87-94.

Pryor, J. Á, \& Webber B.A. (2002). Fisioterapia para problemas respiratórios e cardíacos. (2a ed). Guanabara Koogan.

Silva, R. C. D., Gurian, J. G., Cur, M., Timpone, L. A., \& Judice, M. G., Arantes, A. P. F. (2020). Funcionalidade e qualidade de vida de indivíduos com AVC pós alta da UTI. Rev Insp mov \& saúde, 19(4): 1-18.

Tolep, K, Higgins, N., Muza, S., Griner, G. \& Kelsen, S. G. (1995). Comparison of diaphragm strength between healthy adult elderly and young men. Am J Respir Crit Care Med; 152(2): 677-82.

Viana, D. A., Lilane Maria Alves Silva, L. M. A., Santana, T. C., Ramos, D. A. R., Rodrigues, L. R., \& Carneiro E. M. (2014). Influência dos métodos de predição de peso corporal sobre o volume corrente e mecânica respiratória. Rev. Inspirar.; 6(1):22-26. 